\title{
Antisemitismus unter Geflüchteten aus Syrien und dem Irak. Befunde einer qualitativen Erhebung
}

\author{
Günther Jikeli
}

\section{Einleitung und Forschungsstand ${ }^{1}$}

Die fast 1,5 Millionen Menschen, die von Anfang 2014 bis Juli 2017 in Deutschland einen Erstantrag auf Asyl stellten, sind individuell und nach Herkunft höchst unterschiedlich. Die mit Abstand meisten Anträge stellten in diesem Zeitraum Personen aus Syrien (34 Prozent), Afghanistan (12 Prozent) und dem Irak (10 Prozent). ${ }^{2}$ Von den syrischen Geflüchteten sind 29 Prozent Kurden und 91,5 Prozent muslimischer Herkunft. ${ }^{3}$ SyrerInnen stellen heute in Deutschland nach TürkInnen und PolInnen die drittgrößte Bevölkerungsgruppe mit ausländischem Pass. ${ }^{4}$ Aufgrund der andauernden Kriege und Konflikte, der Zerstörung ganzer Stadtteile und Dörfer in den Herkunftsländern sowie der besseren Lebensperspektiven in Deutschland ist davon auszugehen, dass ein Großteil, vielleicht die Mehrheit der Geflüchteten, auf absehbare Zeit in Deutschland bleiben wird.

Es ist unstrittig, dass antisemitische Vorstellungen in Syrien, Irak und anderen Ländern des Nahen Ostens sowie Nordafrika (MENA) weit verbreitet sind. Umfragen zeigen, dass die große Mehrheit der Bevölkerung antisemitischen Statements zustimmt. In vielen dieser Länder geben über 90 Prozent an, eine negative Meinung über Juden zu haben. ${ }^{5}$

1 Der vorliegende Beitrag ist die überarbeitete und gekürzte Fassung eines Forschungsberichts, der für das American Jewish Committee (AJC) Berlin, Ramer Institute for German-Jewish Relations und die zweite Konferenz des Netzwerks zur Erforschung und Bekämpfung des Antisemitismus (NEBA) erstellt und durch die großzügige Unterstützung des „Bennett Fund“ und des „Meyer Fund“ ermöglicht wurde. (Günther Jikeli, Einstellungen von Geflüchteten aus Syrien und dem Irak zu Integration, Juden und Shoah. Forschungsbericht Dezember 2017, Berlin 2017, [https://ajcberlin.org/sites/default/files/ajc_studie_gefluechtete_und_antisemitismus_2017.pdf], eingesehen 26.10.2018.)

2 Addierte Zahlen aus: Bundesamt für Migration und Flüchtlinge (BAMF), Aktuelle Zablen zu Asyl (2017), Heft 7; BAMF (Hrsg.), Das Bundesamt in Zahlen 2016. Asyl, Migration und Integration, Nürnberg 2017; BAMF (Hrsg.), Das Bundesamt in Zahlen 2014. Asyl, Migration und Integration, Nürnberg 2015. - Von den Personen, die in diesem Zeitraum einen Antrag stellten, befinden sich jedoch viele aufgrund von Rück- und Weiterwanderungen nicht mehr in Deutschland. (Herbert Brücker u. a., IAB-BAMF-SOEP-Befragung von Geflüchteten: Überblick und erste Ergebnisse (Forschungsbericht 29), Nürnberg 2016, S. 18-19.)

3 Zahlen für Erstantragsteller im Jahr 2016. (BAMF (Hrsg.), Das Bundesamt in Zahlen 2016, S. 24-25.)

4 Zahlen für den Stichtag 31.12.2017. (Statistisches Bundesamt, Migration \& Integration, o. D., [https://www.destatis.de/DE/ZahlenFakten/GesellschaftStaat/Bevoelkerung/MigrationIntegration/MigrationInte gration.html], eingesehen 26.10.2018.)

5 Eine Mitte 2013 durchgeführte Umfrage ergab, dass 92 Prozent der Befragten im Irak mindestens sechs der elf abgefragten antisemitischen Aussagen zustimmten. 75 Prozent hielten die Aussage für wahrscheinlich wahr, dass Juden für die meisten Kriege in der Welt verantwortlich seien. 84 Prozent meinten, Juden besäßen zu viel Macht in der Geschäftswelt. (Anti-Defamation League, ADL GLOBAL 100, 2014, [http://global100.adl.org], eingesehen 
Antiisraelische Einstellungen sind nicht weniger verbreitet, wie öffentliche Aufrufe zur Zerstörung Israels und Mobilisierungen gegen eine Normalisierung der Beziehungen mit Israel zeigen. Dies und die Tatsache, dass Antisemitismus, einschließlich Holocaustleugnung, von Regierungsvertretern, islamischen Würdenträgern oder regierungstreuen Medien meist unwidersprochen verbreitet wird, lässt auf eine antisemitische Norm in diesen Ländern schließen. ${ }^{6}$

Die sich als antiimperialistisch, revolutionär und arabisch-nationalistisch verstehende Baath-Partei im Irak betrieb jahrzehntelang antisemitische Propaganda und Hetze gegen Israel und Juden. Die regierende Baath-Partei in Syrien tut dies auch heute noch. Dies wirkte sich auch auf das Bildungssystem und auf die Gestaltung von Schulbüchern aus, die zumindest in Syrien bis heute antizionistische, antisemitische und antiamerikanische Hetze beinhalten. Problematisch ist auch das Geschichtsbild, das syrische Schulbücher von Deutschland vermitteln. Hitler wird hier als starker Führer porträtiert, der sich gegen die Juden zur Wehr setzte, die sich angeblich während der Weltwirtschaftskrise 1929 in Deutschland bereicherten. Juden werden als ewige Feinde der Muslime und Araber sowie der gesamten Menschheit dargestellt. Zionismus und Israel werden in diesen Schulbüchern als imperialistisch und Ursache aller Probleme im Nahen Osten dämonisiert. ${ }^{7}$ Das heißt allerdings nicht, dass alle aus diesen Ländern stammenden Menschen Antisemiten sind, aber diejenigen, die Antisemitismus öffentlich kritisieren, sind in diesen Ländern die Ausnahme.

Dies ist seit der militärischen Niederlage des Nationalsozialismus in Deutschland anders. Offen artikulierter Antisemitismus stößt in der Öffentlichkeit auf Widerspruch und wird im Extremfall, das heißt bei Aufruf zur Gewalt gegen Juden oder bei Verherrlichung des Nationalsozialismus, strafrechtlich geahndet. Ressentiments gegen Juden waren in der deutschen Bevölkerung auch nach 1945 nicht verschwunden, sie wurden aber seither meist über Umwege oder im Privaten geäußert. ${ }^{8}$ Politische Maßnahmen gegen Juden

26.10.2018.) In vielen anderen arabischen Ländern sind die Zahlen laut der ADL-Studie ähnlich. In dem Bericht der im Frühjahr 2009 von PEW erhobenen Umfrage heißt es: „In Arab nations, attitudes toward Jews remain extremely negative. More than $90 \%$ of Egyptians, Jordanians, Lebanese and Palestinians express unfavorable views toward Jews. Only 35\% of Israeli Arabs, however, express a negative opinion.“ (Pew Global Attitudes Project, Little Enthusiasm for Many Muslim Leaders, Washington D.C. 2010, S. 5.)

${ }^{6}$ Zahlreiche Beispiele, die ins Englische übersetzt wurden, können auf der Webseite des Middle East Media Research Institutes eingesehen werden. (MEMRI, 1998-2018, [https://www.memri.org/subjects/antisemitism-documentationproject], eingesehen 26.10.2018.)

7 Siehe u. a. Renate Heugel, Die deutsch-arabische Freundschaft. Deutsche Geschichte (1815-1945) in syrischen Schulbüchern, Hamburg 2013; Arnon Groiss, Jews, Zionism and Israel in Syrian School Textbooks. Report from June 2001, o. O. 2001, [http://www.impact-se.org/wp-content/uploads/2016/04/Syria2001.pdf], eingesehen 26.10.2018; Joshua Landis, Islamic Education in Syria: Undoing Secularism. Prepared for Constructs of Inclusion and Exclusion: Religion and Identity Formation in Middle Eastern School Curricula, Providence (RI) 2003, [http://joshualandis.oucreate.com/Islamic $\% 20$ Education $\% 20$ in $\% 20$ Syria.htm], eingesehen 26.10.2018. - Bezüglich der Schulbücher im Irak, die seit dem Fall Saddam Husseins geändert wurden, siehe Christine Asquith, A New History of Iraq, in: The Guardian, 24.11.2003.

${ }^{8}$ Werner Bergmann/Rainer Erb, Kommunikationslatenz, Moral und öffentliche Meinung. Theoretische Überlegungen zum Antisemitismus in der Bundesrepublik Deutschland, in: KZfSS 38 (1986), Heft 2, S. 223-246. 
wurden jedoch fast ausschließlich von rechtsextremen und linksradikalen Randgruppen gefordert. Dies hat sich sukzessive seit den 1970er-Jahren durch die inzwischen populärste antisemitische Umwegkommunikation in Deutschland, den Antizionismus, geändert. Forderungen nach Maßnahmen gegen den jüdischen Staat, die auf die Einschränkung seiner Fähigkeit hinausläuft, sich effektiv gegen antisemitisch-militante Gruppen zu verteidigen, finden sich heute zumindest vereinzelt bei Politkern aller Parteien. Die „Maßnahmen“ beschränken sich jedoch meist auf öffentliche Verurteilungen Israels und stehen engen Kooperationen auf wissenschaftlichem und kulturellem Gebiet gegenüber - wie auch einer „Staatsräson“, nach der für Deutschland das „Existenzrecht Israels unverhandelbar“ sei. ${ }^{9}$ Die öffentlichen Pauschalverurteilungen von Israel im Namen der Menschenrechte, die eben keine Kritik an bestimmten Regierungsentscheidungen sind, blenden den terroristischen Charakter von Hamas und anderen palästinensischen Gruppen aus, die zum Krieg gegen Israel und die Juden nicht nur aufrufen, sondern dies in die Tat umsetzen. ${ }^{10}$ Sie sind auch oft verbunden mit der Verwendung klassischer antisemitischer Stereotype wie ,jüdischer Rachsucht“ oder dem alten Ritualmordvorwurf in Form der Anschuldigung, (palästinensische) Kinder mit Absicht oder gar aus Lust zu töten. Darüber hinaus wirkt sich die Dämonisierung Israels direkt auf Jüdinnen und Juden in Deutschland aus, denn sie stehen unter Generalverdacht, Sympathien für den angeblich menschenverachtenden Staat Israel zu hegen und werden dafür angefeindet. Dabei ist es unerheblich, wie Jüdinnen und Juden in Deutschland zu Israel stehen, auch wenn es zutrifft, dass sich viele Jüdinnen und Juden aus historischen, religiösen und familiären Gründen mit Israel verbunden fühlen.

Vor diesem Hintergrund, der antisemitischen und antiisraelischen Norm in Ländern des Nahen Ostens und Nordafrika, einer weiten Verbreitung von antiisraelischen Einstellungen in der deutschen Bevölkerung, die meist mit Ressentiments gegen Jüdinnen und Juden einhergehen, sowie einer Häufung von öffentlich gewordenen antisemitischen Vorfällen, ist es nicht verwunderlich, dass VertreterInnen jüdischer Gemeinden Sorge über eine Zunahme von Antisemitismus auch aufgrund der

\footnotetext{
9 Bundeskanzlerin Angela Merkel bezeichnete schon 2006 während ihrer Reise in die USA das Eintreten für das Existenzrecht Israels als „unverrückbare Konstante deutscher Außenpolitik“ und betonte 2008 vor der Knesset: „die Sicherheit Israels ist für mich als deutsche Bundeskanzlerin niemals verhandelbar." Prinzipiell wird dies auch 2018 von allen im Bundestag vertretenen Parteien anerkannt, wie entsprechende Anträge im April 2018 zum 70. Jahrestag der Gründung des Staates Israel zeigen. (Siehe u. a. Deutscher Bundestag, Drucksache 19/1823, 24.4.2018, [http://dipbt.bundestag.de/doc/btd/19/018/1901823.pdf], eingesehen 26.10.2018; Deutscher Bundestag, Drucksache 19/1850, 25.4.2018, [https://dipbt.bundestag.de/doc/btd/19/018/1901850.pdf], eingesehen 26.10.2018.) - Doch bereits diese Formulierung weist auf ein gespanntes Verhältnis hin, denn es steht keineswegs im rechtlichen oder moralischen Kompetenzrahmen der Bundesrepublik, darüber zu befinden, ob Israel ein Existenzrecht hat oder nicht. Dass mit der Formel von der „Anerkennung des Existenzrechts“ keine militärische, finanzielle oder diplomatische Solidarisierung gemeint ist, wird beispielweise angesichts des Abstimmungsverhaltens Deutschlands in den Vereinten Nationen in Bezug auf einseitige Verurteilungen Israels oder auch angesichts der mangelnden Reaktion gegenüber wiederholten Vernichtungsdrohungen gegen Israel seitens des iranischen Regimes deutlich.

10 Alex Feuerherd/Florian Markl, Vereinte Nationen gegen Israel. Wie die UNO den jüdischen Staat delegitimiert, Berlin 2018, S. 193-219.
} 
Günther Jikeli

Zuwanderung hunderttausender Menschen aus Ländern wie Syrien, Irak und Afghanistan äußern. Eine Umfrage aus dem Jahr 2016 belegt, dass viele Jüdinnen und Juden sich vor antisemitischen Angriffen fürchten. ${ }^{11}$ Entsprechende Befürchtungen wurden bestärkt, als es im Dezember 2017 auf einer antiisraelischen Demonstration in Berlin zu antisemitischen Sprechchören in arabischer Sprache und zur Verbrennung einer israelischen Fahne kam, ${ }^{12}$ und im April 2018, als ein kippatragender Jugendlicher in Berlin von einem Geflüchteten aus Syrien beschimpft und geschlagen wurde. ${ }^{13}$ Im Juli 2018 wurde über einen antisemitischen Vorfall berichtet, bei dem ein Syrer, der aufgrund eines Kettenanhängers als Jude identifiziert wurde, von einer Gruppe junger Menschen zusammengeschlagen wurde, unter denen sich sechs Syrer befanden. ${ }^{14}$ Das Problem wird inzwischen auch von vielen PolitikerInnen und RegierungsvertreterInnen verschiedener Parteien erkannt, ${ }^{15}$ zugleich jedoch für pauschale Polemisierungen gegen Geflüchtete benutzt. Die Thematisierung von Antisemitismus unter Geflüchteten findet in einem gesellschaftlichen Rahmen statt, in dem zwar auf der einen Seite nach wie vor große Teile der deutschen Öffentlichkeit Sympathien gegenüber Geflüchteten bekunden und Integrations- und Hilfsprogrammen für Geflüchtete unterstützen, auf der anderen Seite aber ein nicht unbeträchtlicher Teil der Bevölkerung neuen (und alten) Migranten ablehnend gegenüber steht. Das zeigt eindrücklich die hohe Anzahl von Angriffen gegen

${ }^{11}$ Eine Befragung der Universität Bielefeld im Auftrag des vom Bundestag berufenen Unabhängigen Expertenkreises Antisemitismus im Mai und Juni 2016 ergab, dass sich 37 Prozent der befragten Jüdinnen und Juden besorgt vor körperlichen Übergriffen äußerten und 58 Prozent der Befragten aus Sicherheitsgründen bestimmte Stadtteile oder Orte vermeiden. 70 Prozent tragen aufgrund erwarteter Gefahren keine äußerlich erkennbaren jüdischen Symbole. (Andreas Zick u. a., Jüdische Perspektiven auf Antisemitismus in Deutschland. Ein Studienbericht für den Expertenrat Antisemitismus, Bielefeld 2017, S. 4.)

12 Demonstranten verbrennen israelische Flaggen in Berlin, in: Die Welt, 9.12.2017, [https://www.welt.de/politik/deutschland/article171430290/Demonstranten-verbrennen-israelische-Flaggen-inBerlin.html], eingesehen 26.10.2018. - Anlass der Demonstration war die Erklärung der amerikanischen Regierung, Jerusalem als Hauptstadt Israels anzuerkennen. Ob Geflüchtete an der Demonstration beteiligt waren ist allerdings nicht bekannt - mobilisiert wurde vor allem von der Muslimbruderschaft nahestehenden Organisationen. Der Rat der Imame und Gelehrten in Deutschland (RiGD), der mit dem Zentralrat der Muslime assoziiert ist, rief dazu auf, „Jerusalem als Besitz der Muslime zu betonen“. „Der östliche wie der westliche Teil Jerusalems sind als arabisches muslimisches Land zu betrachten, das nicht alleiniger Besitz der Palästinenser ist, sondern Besitz aller Muslime weltweit", hieß es weiterhin in einer Stellungnahme. (RiGD, Pressemitteilung vom 7.12.2017,

[https://www.facebook.com/698425836929834/photos/a.786083888164028.1073741829.698425836929834/13249 48617610883], eingesehen 26.12.2018.)

13 Der Vorfall ereignete sich am 17.4.2018 in Prenzlauer Berg (Berlin). Das Opfer, das beschimpft und mit einem Gürtel geschlagen wurde, war ein arabischer, nicht-jüdischer Israeli, der den Beweis antreten wollte, dass es ungefährlich sei, sichtbar mit Kippa, durch Berlin zu spazieren. Zahlreiche Medien berichteten über diesen Vorfall.

14 Attacke in Berlin - Gruppe prügelt auf Syrer ein, in: Frankfurter Rundschau, 7.7.2018.

${ }^{15}$ Die ehemalige Bundesjustizministerin Katarina Barley (SPD) nannte gegenüber Der Welt den „Flüchtlingszustrom“ als eine der Ursachen des Anstiegs des Antisemitismus. (Die Welt, 21.4.2018.) - Der Tagesspiegel zitierte die Bundeskanzlerin im April 2018 aus einer Stellungnahme gegenüber dem israelischen Nachrichtensender Channel 10 News: „Wir haben jetzt auch neue Phänomene, indem wir Flüchtlinge haben oder Menschen arabischen Ursprungs, die wieder eine andere Form von Antisemitismus ins Land bringen." (Der Tagesspiegel, 22.4.2018.) 
Geflüchtete und deren Unterkünfte, selbst wenn Kriminalstatistiken belegen, dass Geflüchtete auch häufig Opfer von Straftaten anderer MigrantInnen werden. ${ }^{16}$

Die hier vorgestellte (siehe unten) sowie zwei weitere empirische, aber nicht repräsentative Studien bestätigen, dass zumindest unter den interviewten Geflüchteten antisemitische und antiisraelische Vorstellungen weit verbreitet sind, dass es jedoch deutliche Unterschiede zwischen diversen religiösen und ethnischen Gruppen gibt.

Bei einer Umfrage aus Bayern unter 779 Geflüchteten aus Syrien, dem Irak, Eritrea und Afghanistan stimmten 52 Prozent der syrischen und 54 Prozent der irakischen Befragten der Aussage ,Juden haben auf der Welt zu viel Einfluss“ zu. Unter Afghanen waren dies 57 Prozent und unter Eritreern 5 Prozent. In der deutschen Bevölkerung schwanken die Zustimmungsraten zu dieser oder ähnlichen Aussagen in den letzten Jahren zwischen 15 und 25 Prozent. ${ }^{17}$ Das Ausmaß der Zustimmung ist in der Umfrage unter Geflüchteten nicht abhängig von Variablen wie Alter, Familienstand, Bildungsniveau, der bisherigen Aufenthaltsdauer in Deutschland oder dem Grad der Religiosität, wohl aber von der Religionszugehörigkeit. Muslime stimmten der Aussage mit über fünfzig Prozent deutlich häufiger zu als Christen mit 22 Prozent. Im Rahmen derselben Studie wurden auch qualitative Interviews mit zwölf Geflüchteten, darunter vier aus Syrien und vier aus dem Irak, geführt. Darin äußerten sich die Befragten nicht antisemitisch, verwiesen aber auf judenfeindliche Einstellungen in ihren jeweiligen Herkunftsländern. ${ }^{18}$ In einer anderen qualitativen Studie, in der 25 Geflüchtete aus Syrien, Afghanistan und dem Irak nach ihren Einstellungen zu Juden befragt wurden, stellen die Autorinnen einen „AlltagsAntizionismus“ fest, „der Israel für alle negativen Entwicklungen in der Region verantwortlich macht", sowie negative Einstellungen gegenüber Juden, die als selbstverständlich erachtet werden. ${ }^{19}$ Die Mehrheit der Befragten in dieser Studie äußerte sich antisemitisch, eine Interviewpartnerin sagte sogar explizit, sie hasse Juden.

Eine Studie des Londoner Pears Instituts im Auftrag der Stiftung Erinnerung, Verantwortung und Zukunft (EVZ) stellt in Bezug auf die Zuwanderung seit 2011 aus der MENA-Region (Länder des Nahen Ostens) keinen solchen Zusammenhang fest. ${ }^{20}$ Sie verwendet allerdings Methoden, mit der ein solcher auch gar nicht festgestellt werden

${ }^{16}$ Eine Chronik flüchtlingsfeindlicher Vorfälle ist auf einer gemeinsamen Webseite der Amadeu Antonio Stiftung und Pro Asyl einsehbar. (Mut gegen rechte Gewalt, o D., [https://www.mut-gegen-rechte-gewalt.de/service/chronikvorfaelle], eingesehen 26.10.2018.)

${ }_{17}$ Bericht des Unabhängigen Expertenkreises Antisemitismus, 7.4.2017, Deutscher Bundestag, Drucksache 18/11970, S. 63, [http://dip21.bundestag.de/dip21/btd/18/119/1811970.pdf], eingesehen 26.10.2018.; Anti-Defamation League, ADL GLOBAL 100.

18 Sonja Haug u. a., Asylsuchende in Bayern. Eine quantitative und qualitative Studie. Abschlussbericht, München 2017, S. 66-69.

19 Sina Arnold/Jana König, Flucht und Antisemitismus. Erste Hinweise zu Erscheinungsformen von Antisemitismus bei Geflüchteten und mögliche Umgangsstrategien. Qualitative Befragung von Expert_innen und Geflüchteten, Berlin 2016, S. 29.

20 Die Studie besteht aus fünf Länderberichten (Belgien, Deutschland, Frankreich, Großbritannien und Niederlande) und einem Gesamtbericht. (David Feldman: Antisemitismus und Immigration im heutigen Westeuropa. Gibt es einen Zusammenhang? Ergebnisse und Empfehlungen einer Studie aus fünf Ländern, Berlin-London 2018.) 
kann, geschweige denn das Gefahrenpotenzial, das möglicherweise von antisemitisch eingestellten Zuwanderern ausgeht. Mit Hilfe repräsentativer Umfragen soll belegt werden, dass es durch den Anstieg der Migration seit 2011 nicht zu einem Anstieg von Antisemitismus in Deutschland gekommen ist. Dies ist allerdings unsinnig, denn die Gruppe der neuen MigrantInnen, die nach 2011 nach Deutschland gekommen ist (insgesamt etwas über 1,5 Millionen Menschen und davon nur ein Teil aus der MENARegion) ist bei 83 Millionen Menschen in Deutschland zu gering, um in den für die Gesamtbevölkerung repräsentativen Umfragen erfasst zu werden. Die Gruppe der neuen MigrantInnen liegt prozentual deutlich unter der in Umfragen verwendeten statistischen Fehlervarianz zwischen drei und fünf Prozent. Das heißt, selbst wenn alle neuen MigrantInnen sich antisemitisch äußern würden, wäre das in den repräsentativen Umfragen nicht nachweisbar, wenn nicht speziell nach dieser Gruppe differenziert wird. Dass auch von einer relativ kleinen Gruppe eine Gefahr jetzt oder in Zukunft ausgehen kann, wenn besonders starke antisemitische Einstellungen vorhanden sind, wird nicht erörtert. Erstaunlicherweise zitiert der Autor für den Bericht für Deutschland zwar die von Julia Bernstein geleitete Umfrage unter jüdischen Betroffenen, der zufolge 81 Prozent der Befragten angaben, dass körperliche Angriffe von Muslimen ausgingen. Bei Beleidigungen oder Belästigungen waren dies 62 Prozent. Er kommt aber dennoch zu dem Schluss, dass „Täter immer noch vornehmlich nicht-Migranten, nicht-muslimische Deutsche mit einen rechtsextremen Hintergrund sind."21 Grundlage für diese Behauptung sind Polizei- und Verfassungsschutzstatistiken, die einen muslimischen Hintergrund gar nicht erfassen und beispielsweise Hakenkreuzschmierereien automatisch als rechtsextrem einordnen. ${ }^{22}$ Auch deshalb werden derzeit die Erfassungssysteme von antisemitischen Vorfällen reformiert, beziehungsweise neu aufgebaut. Die Datenlage ist zumindest nicht eindeutig und es ist unersichtlich, warum der Autor in diesem Punkt die Betroffenenperspektive in seiner Zusammenfassung unter den Tisch fallen lässt.

Im von David Feldman verfassten Gesamtbericht heißt es, im Widerspruch zum Teilbericht Deutschland, dass in Deutschland antisemitische Einstellungen insgesamt eher abnähmen. ${ }^{23}$ Während einige Umfragen eine Abnahme konstatieren, verweisen andere auf eine Zunahme ab 2014 - oder eine Fluktuation auf hohem Niveau. In Umfragen im Auftrag der Anti-Defamation League sind die Zustimmungsraten zur Aussage ,Juden haben zuviel Macht in der Geschäftswelt," seit 2002 wie folgt: Mai und September 2002: 32\%, März 2004: 24\%, April 2005: 20\%, März 2007: 21\%, Dezember 2008 bis Januar 2009: 21\%, Januar 2012: 22\%, August bis September 2013: 33\%, MärzApril 2015: 28\%. ${ }^{24}$ Ähnlich sieht es aus bei der Aussage ,Juden sprechen noch immer

21 Mathias Berek, Antisemitism and Immigration in Western Europe Today. Is there a connection? The case of Germany, Berlin-London 2018, S. 7.

22 Ist die Statistik zu antisemitischen Straftaten irreführend?, in: Badische Zeitung, 22.3.2018.

${ }^{23}$ David Feldman, Antisemitismus und Immigration im heutigen Westeuropa, S. 19 und 31.

${ }^{24}$ Siehe die Umfrageberichte ADL, European Attitudes Toward Jews, Israel and the Palestinian-Israeli Conflict, Juni 
zuviel über den Holocaust.“ Hier stimmten 2015 immerhin 51 Prozent zu. Eine Zu- oder Abnahme des Antisemitismus lässt sich jedoch nicht allein mit Einstellungsumfragen beurteilen. Zwei Umfragen unter Betroffenen werden zwar genannt, aber dann in den Schlussfolgerungen schlicht ignoriert: In der eingangs erwähnten Studie der Universität Bielefeld gaben 201678 Prozent der befragten Jüdinnen und Juden an, dass der Antisemitismus in den letzten fünf Jahren zugenommen habe. ${ }^{25}$ Ähnliches ergab schon eine Studie der Agentur der Europäischen Union für Grundrechte (FRA), nach der 2012 68 Prozent der befragten Jüdinnen und Juden in Deutschland eine Zunahme des Antisemitismus in den letzten fünf Jahren wahrnahmen. ${ }^{26}$

Die Daten des Bundeskriminalamts (wenn auch hinsichtlich der Tätergruppen unzuverlässig) zeigen keineswegs eine Abnahme der registrierten antisemitischen Straftaten seit 2011, sondern einen leichten Anstieg mit einem Peak jeweils 2012 und $2014 .^{27}$

Die Schlussfolgerungen der Studie des Londoner Pears Instituts sind wohl eher als politische Meinungsäußerung zu werten und nicht als Ergebnis fundierter Forschung. Schwerer wiegt vielleicht aber, dass der Gesamtbericht schlussfolgernd behauptet, dass der Antisemitismus abnehme. Einschlägige Umfragen unter jüdischen Betroffenen, die besagen, dass Antisemitismus in den letzten Jahren zugenommen habe und dass ein Großteil der Täter einen muslimischen Hintergrund habe, finden keine Berücksichtigung als Indikator für die weite Verbreitung von Antisemitismus. Implizit wird damit die Perspektive der Betroffenen delegitimiert. Dazu passt, dass Repräsentanten jüdischer Gemeinden, die vor Antisemitismus unter neuen MigrantInnen warnen, im öffentlichen Diskurs und in der EVZ-Studie häufig in einem Atemzug mit rechtspopulistischen Politikern genannt werden. Dies zeigt einmal mehr, wie sehr das Thema Antisemitismus unter (geflüchteten) Muslimen selbst in der Wissenschaft ideologisiert wird und wie wichtig es ist, der Fragestellung mit angemessenen Methoden zu begegnen und entsprechendes empirisches Material zu erheben.

2002; dies., European Attitudes Toward Jews. A Five Country Survey, August 2002; dies., Attitudes Toward Jews, Israel and the Palestinian-Israeli Conflict in Ten European Countries, August 2004; dies., Attitudes Toward Jews in Twelve European Countries, May 2005; dies., Attitudes Toward Jews and the Middle East in Five European Countries, May 2007; dies., Attitudes Toward Jews in Seven European Countries, February 2009; dies., Attitudes Toward Jews In Ten European Countries, March 2012; dies., ADL GLOBAL 100. 2015 Update Germany, o. D. [http://global100.adl.org/\#country/germany/2015], eingesehen 7.11.2018.

${ }_{25}$ Zick, Jüdische Perspektiven auf Antisemitismus, S. 13.

${ }^{26}$ European Union Agency for Fundamental Rights, Discrimination and hate crime against Jews in EU Member States: experiences and perceptions of antisemitism, Wien 2013, S. 17.

27 Siehe hierzu die Verfassungsschutzberichte der Jahre 2001 bis 2017. (Bundesamt für Verfassungsschutz, o. D., [https://www.verfassungsschutz.de/de/oeffentlichkeitsarbeit/publikationen/verfassungsschutzberichte], eingesehen 28.10.2018.) 


\section{Methoden der Studie und Sample}

Die hier vorliegende Studie hat keinen Anspruch auf Repräsentativität, sondern es werden vielmehr wiederkehrende Muster von Bildern über Jüdinnen und Juden untersucht: Welche Stereotype und Vorstellungen gibt es? Welche davon haben spezifische Bezüge zum jeweiligen Hintergrund der Zielgruppe? Welche Begründungsmuster und Zusammenhänge lassen sich feststellen?

Im Dezember 2016 wurden in Berlin in 16 Gruppeninterviews insgesamt 68 Geflüchtete aus Syrien und dem Irak befragt. Die Interviews dauerten durchschnittlich etwa neunzig Minuten und wurden mithilfe von drei Übersetzern und einer Übersetzerin geführt, die selbst Geflüchtete aus Syrien sind: einer muslimisch-arabischen Frau sowie einem christlich-arabischen Mann, einem muslimisch-arabischen und einem muslimischkurdischen Mann. Die Gespräche fanden, bis auf ein Gespräch auf Kurdisch und zwei auf Englisch, alle auf Arabisch statt. Teile der Gespräche wurden zudem auf Deutsch geführt. Fragen des Interviewers wurden ins Arabische beziehungsweise Kurdische sowie die Antworten der Befragten ins Englische übersetzt. Das Sample umfasst separate Gruppen von Geflüchteten: Geflüchtete über 27 Jahren, Geflüchtete unter 27 Jahren, Frauen, Männer, AraberInnen, KurdInnen, PalästinenserInnen (aus Syrien) sowie gemischte Gruppen. Alle Interviews wurden vom Autor geführt, der sich als Wissenschaftler der Indiana University und der Universität Potsdam vorstellte.

Die Interviewten wurden zum einen über Beschäftigte in der Flüchtlingsarbeit gewonnen und zum anderen über die vier ÜbersetzerInnen beziehungsweise deren Bekannte. Um nicht nur intrinsisch motivierte Personen $\mathrm{zu}$ befragen, wurde mit einer Aufwandsentschädigung von zwanzig Euro ein zusätzlicher Anreiz für die Teilnahme geschaffen. Laut der vermittelnden SozialarbeiterInnen gehört ein Großteil der Befragten zu den eher „aufgeschlossenen und toleranten“ Geflüchteten, die sich besonders um Integration und Kontakt mit der deutschen Bevölkerung bemühen. Erschwerend kommt hinzu, dass eine vom Staat unabhängige Forschung in den diktatorischen Regimen der Herkunftsländer unüblich ist und die Interviewten im Asylverfahren, das ebenfalls ein „Interview“ beinhaltet, stark abhängig vom deutschen Staat sind. Dies mag dazu geführt haben, dass Personen, die vermuten, dass sie sozial unerwünschte Einstellungen wie beispielsweise Islamismus und offener Judenhass teilen, im Sample unterrepräsentiert sind. ${ }^{28}$

${ }^{28}$ Für ein Folgeprojekt interviewte der Autor gemeinsam mit drei Wissenschaftlern der Indiana University, Haidar Khezri, Asaad al Saleh und Alvin Rosenfeld, im Sommer 2017 in Berlin, Greifswald und Köln weitere 85 Geflüchtete aus Syrien und dem Irak sowie zwanzig in der Flüchtlingsarbeit beschäftigte Personen. Diese ausführlicheren und größtenteils individuell geführten Interviews sind nicht Gegenstand dieses Berichts. Eine grobe Auswertung zeigt aber, dass die Interviews des Folgeprojekts den hier dargelegten Befunden nicht widersprechen. In einigen Bereichen, insbesondere in Bezug auf Einstellungen zu Juden, ist aber eine größere Vielfalt feststellbar, einschließlich radikalen, offen genozidalen Einstellungen. 
Einige der angesprochenen Themen wie islamistischer Terrorismus, Geschlechtergerechtigkeit, der Nahhostkonflikt oder Juden und Israel gelten als sensibel und der Interviewer und die ÜbersetzerInnen stießen folglich teilweise auf Zurückhaltung, Ablehnung und Misstrauen. Die zu Beginn des Interviews offengelegte Verbindung des Interviewers $\mathrm{zu}$ einer amerikanischen Universität mag darüber hinaus bei antiamerikanisch eingestellten Personen zu Zurückhaltung geführt haben. Möglicherweise wurden daher eventuell vorhandene antidemokratische und speziell antisemitische und antiamerikanische Einstellungen nicht offen im Interview geäußert. Zwei Gruppen wussten vor dem Interview, dass ein zentrales Thema Einstellungen zu Juden ist: offen antijüdische Einstellungen wurden in diesen Interviews nicht geäußert. Die übrigen interviewten Personen wussten im Vorfeld, dass die Interviews Teil einer wissenschaftlichen Befragung zu Einstellungen unter Geflüchteten in Deutschland waren.

Die Interviews wurden in ruhigen Cafés, Restaurants oder in verschiedenen Berliner Gemeinschaftsunterkünften, in Seminarräumen oder in den Zimmern der Befragten, durchgeführt. Im Anschluss an die Interviews wurden die Befragten gebeten, einen kurzen Fragebogen zu biografischen Daten auszufüllen (Alter, Beruf, Bildungsstand, Geburtsort, Religion, ethnischer Hintergrund, Sprachkenntnisse, derzeitige Beschäftigung, Aufenthaltsdauer in Deutschland). Die Interviews wurden aufgezeichnet, vollständig übersetzt und transkribiert. Die Auswertung erfolgte in Zusammenarbeit mit Dr. Lars Breuer und Dr. Matthias J. Becker anhand einer induktiven und deduktiven Kodierung der Interviews mithilfe der Auswertungssoftware MAXQDA in einem iterativen Prozess der Kategorienbildung.

Alle Interviewten, 14 Frauen und 54 Männer im Alter von 18 bis 52 Jahren, erreichten Deutschland seit Mitte 2014, die meisten kamen im Verlauf des Jahres 2015. 55 Geflüchtete identifizierten sich selbst als AraberInnen, davon elf als arabisch/syrischpalästinensisch $^{29}$ und 13 als KurdInnen aus Syrien. Die überwiegende Mehrheit ist muslimischen Glaubens, sechs bezeichneten sich als atheistisch (eine davon mit christlichem Hintergrund), vier als christlich und einer als Druse. Das Bildungsniveau und die Religiosität variierten erheblich. Die Befragten stammten aus kleineren und größeren Städten wie Aleppo, Bagdad, Damaskus, Hama, Homs, Idlib, Qamlishi und Raqqa oder deren Umgebung. Insgesamt hatten die Befragten ein positives Bild von der deutschen Bevölkerung. Ein Gefühl des Willkommenseins, zumindest am Anfang, war bei vielen verbreitet. Dies entspricht dem Empfinden einer Mehrheit der Geflüchteten in Deutschland, wie eine repräsentative Studie zeigt. ${ }^{30}$ Viele berichteten jedoch auch von Problemen, etwa der Sorge um den Aufenthaltstitel, Schwierigkeiten bei der Familienzusammenführung, der Suche nach einer Wohnung, dem Erlernen der deutschen Sprache, der Anerkennung von Papieren, diversen Behördengängen oder der

${ }^{29}$ Dabei handelt es sich um Personen, die in Syrien aufgewachsen sind.

${ }^{30}$ Brücker, IAB-BAMF-SOEP-Befragung, S. 32-36. 
Suche nach Arbeit beziehungsweise geeigneten Qualifizierungsmaßnahmen. Auch die Sorge um Familienangehörige im Herkunftsland spielten bei vielen Befragten eine große Rolle. Insbesondere die als undurchsichtig erlebte Bürokratie der Sozialämter und Ausländerbehörden sowie die Unterbringung in Gemeinschaftsunterkünften führten häufig zu Frustrationen. ${ }^{31}$ Die allermeisten Geflüchteten strebten eine Integration im Sinne gesellschaftlicher Teilhabe, Arbeitsaufnahme und Respektierung der Gesetze an. Unter den Jüngeren galt als selbstverständlich, dass dazu auch das Erlernen der deutschen Sprache gehört, auch wenn den allermeisten die Beibehaltung ihrer ethnischen Identität sowie ihrer Werte in Bezug auf Familie und Religion sehr wichtig war.

\section{Identitäten: Arabischer Nationalismus und Positionierungen gegen Jihadismus}

Keiner der Interviewten zeigte offene Sympathien für islamistische oder terroristischislamistische Gruppen. „Extremismus“ und „Terrorismus“ wurden weit von sich gewiesen und meist auch vom Islam an sich. Die Leugnung jeglicher Verbindung von Jihadisten zum Islam kann jedoch schnell in Verschwörungstheorien enden. Islamistischterroristische Gruppen wie der „Islamische Staat" (IS) und „al-Qaida“ wurden als unislamisch definiert. Zugespitzt kann diese Argumentationsstrategie dazu führen, dass andere, vorzugsweise Juden, Israel oder die USA, für islamistische Terrorgruppen verantwortlich gemacht werden: „Daesch [arabische Abkürzung für den IS; Anm. GJ] hat in keiner Weise etwas mit dem Islam zu tun [...]. Amerika hat Daesch geschaffen." (Sayyid, 21, arabisch-muslimischer Mann aus Raqqa/Syrien) ${ }^{32}$ Die Gruppe der fünf Interviewten war sich einig, dass Amerika die Anschläge auf das World Trade Center in New York am 11. September 2001 verübt hatte, und dass ,auch Juden etwas damit zu tun haben, da kein jüdischer oder israelischer Angestellter in dem Gebäude war.“ (Sayyid) Amerika und „die Juden" wurden für das Handeln islamistisch-terroristischer Organisationen verantwortlich gemacht und jegliche Verbindung islamistischer Gruppen zum Islam wurde geleugnet. Einige Befragte machten anstelle von Juden auch Israel verantwortlich. Für Yanes (32, arabisch/palästinensisch-muslimischer Mann aus Damaskus/Syrien) war der IS nichts weiter als eine Marionette der USA und Israels.

Unter weniger religiösen Interviewten fanden sich dagegen auch deutlichere Stimmen, die von „islamischen Extremisten“ sprachen und diese durchaus als eigenständige, gefährliche Akteure betrachteten, die es zu bekämpfen gilt. Aber auch unter den weniger Religiösen tendierten viele Befragte dazu, terroristisch-islamistischen Gruppen ihre islamische Identität abzusprechen. Dies führte zum Teil zu widersinnigen Behauptungen.

31 Die repräsentative IAB-BAMF-SOEP-Befragung zeigt ebenfalls eine relative Unzufriedenheit über die Wohnsituation in Gemeinschaftsunterkünften im Vergleich zu Privatwohnungen. (Ebd.)

32 Bei den angegebenen Namen der interviewten Personen handelt es sich um Pseudonyme. 
So meinte etwa die liberale, säkulare und ismailitische ${ }^{33}$ Aktivistin Tarana: „ISIS, das sind keine Muslime, [...] die sind Teil des [syrischen] Regimes." Sie sah die Kriege im Nahen Osten als Teil eines großen Plans, in dem die israelische Lobby die USA beherrsche. Erbitterte Gegner im syrischen Bürgerkrieg sind darin Teil derselben Macht und Muslime können gemäß dieses Narrativs keine Terroristen sein.

Arabische Interviewte waren oft stolz auf ihre arabische Identität und verorteten den Ursprung der menschlichen Zivilisation in Syrien und im Irak. Im Extremfall kamen dabei rassistische Gefühle der Überlegenheit und Abwertung von anderen zum Ausdruck. Diese Überlegenheitsgefühle eines arabischen Nationalstolzes werten nicht nur andere Menschen $a b$, sondern dienten paradoxerweise als Erklärung für das derzeitige Scheitern der arabischen Nationalstaaten Syrien und Irak. Die eigene Nation wurde als ganz besonders potent imaginiert, weshalb sie Ziel von Angriffen und Verschwörungen sei.

\section{Bilder von Jüdinnen und Juden: Von „Eine Religion wie unsere“ bis zu Weltverschwörern}

In den Interviews fand sich eine große Bandbreite von Einstellungen gegenüber Jüdinnen und Juden: Diese reichte von pro-jüdischen und pro-israelischen Bekenntnissen über Toleranz, Desinteresse, einzelne Stereotype bis hin zu antisemitischen Weltverschwörungsphantasien und genozidalen Einstellungen, die sich unter anderem in der Rechtfertigung der Shoah ausdrückten. Auffallend war dabei die Diskrepanz zwischen Angehörigen der Mehrheitsbevölkerung im jeweiligen Herkunftsland und den Angehörigen der jeweiligen ethnischen und religiösen Minderheiten. Insbesondere einige der interviewten syrischen KurdInnen zeigten deutlich pro-jüdische und pro-israelische Einstellungen. Teilweise waren diese jedoch philosemitisch durchsetzt, das heißt Juden wurden für ihre Macht und Cleverness bewundert. Einige der Interviewten mit syrischpalästinensischem Hintergrund zeigten sich hingegen besonders antisemitisch: sowohl in Bezug auf Israel als auch Verschwörungsphantasien.

Positiv zu vermerken sind vereinzelte Aussagen, die auf ein Aufbrechen des Feindbildes Israel verweisen: Einige Befragte sahen Israel im Vergleich zum IS oder zum syrischen Regime als deutlich humaner. Dies war freilich nur dann der Fall, wenn diese Personen nicht davon ausgingen, dass Israel für die Kriege im Irak und in Syrien verantwortlich ist. Jüdinnen und Juden wurden primär als religiöse Gemeinschaft und nicht als „Volk“ wahrgenommen. Letzteres wurde ausgeblendet oder explizit geleugnet und war eng verbunden mit der Ablehnung des Staates Israel als jüdischer Staat. Vor allem aber zeigten die Interviews, dass die allermeisten Befragten trotz verbreiteter

${ }^{33}$ Die schiitische Glaubensgemeinschaft der Ismailiten gilt als tolerant und offen. Sie wird von sunnitischen Muslimen oft als abtrünnig angesehen. 
verschwörungstheoretisch-antisemitischer Denkweisen zur Zeit des Interviews mit anderen Dingen beschäftigt waren, sodass Jüdinnen und Juden keinen zentralen Platz in ihrer unmittelbaren Gedankenwelt einnahmen.

Antisemitismus brach meist erst auf Nachfragen zu Themen wie Ursachen für Kriege im Nahen Osten, islamistische terroristische Angriffe wie am 11. September 2001, Ursachen für die Entstehung des IS, vorherrschende Bilder von Israel, die Shoah oder die Rolle von Juden vor dem Zweiten Weltkrieg in Deutschland hervor. Spontan wurden judenfeindliche Bilder im Zusammenhang mit der Erwähnung von Freimaurern, der Familie Rothschild, Zionismus sowie Banken und Finanzmärkten genannt. In Diskussionen zu traditionellen Beziehungen zwischen Juden und Muslimen fiel ein ambivalentes Verhältnis auf, in dem zum einen ein angebliches harmonisches Miteinander sowie religiöse Toleranz betont und zum anderen eine tiefgehende immerwährende Feindschaft zwischen Juden und Muslimen suggeriert wurde - teilweise von denselben Personen.

\section{Antisemitismus und Religion: Islamischer Antisemitismus}

Explizit religiös begründeter Antisemitismus wurde im Vergleich zu anderen Begründungen relativ selten geäußert. Generell waren die muslimischen Befragten darum bemüht, ein positives Bild des Islam zu zeichnen und diesen nicht als Ursache von potentiellen Konflikten darzustellen. Dennoch gaben die Interviewten auch Vorstellungen wieder, die islamisch-antijüdische Stereotype bedienen. Darunter fallen die Ansichten, dass Juden und Muslime generell Feinde seien, dass „die Juden“ die heiligen Schriften verfälscht hätten und dass sie versucht hätten, den Propheten Mohammed zu vergiften. ${ }^{34}$ Auch positive Stereotype begründeten Befragte mit Verweisen auf islamische Quellen: ,Juden sind fähig, Juden sind die Söhne Gottes, Juden ... das ist der Grund, warum sie Geld haben und der Koran besagt, dass sie hart arbeiten." (Nidal, 35, kurdischer Mann aus Aleppo/Syrien mit muslimischem Hintergrund)

Die Annahme einer generellen Feindschaft zwischen „den Juden“ und „den Muslimen“ hat ein radikales Potenzial, da sie essentialistisch auf die Gesamtheit der Jüdinnen und Juden, einschließlich in Deutschland, bezogen wird. Ein Beispiel ist die Reaktion von Yanes auf eine Äußerung Jalils, der in einem Gespräch über das Zusammenleben von Menschen unterschiedlicher Religionen in Deutschland betonte, dass der Islam eine friedliche Religion sei und dass ,unser Prophet Mohammed uns gesagt hat, dass wir alle

\footnotetext{
34 Entsprechende Interpretationen finden sich in vielen Strömungen des Islam. Der Koran und die Hadithen beschreiben die Beziehungen zwischen Juden und Muslimen ambivalent. Als Beleg einer grundsätzlichen Feindschaft werden verschiedene Stellen im Koran herangezogen, darunter: Sure 5, Vers 82; Sure 2, Vers 61 und Vers 96, sowie Sure 9, Vers 29. Der Vorwurf der Fälschung heiliger Schriften findet sich in Sure 4, Vers 46 und Sure 2, Vers 75. Eine Geschichte, in der eine Jüdin versucht habe, Mohammed zu ermorden findet sich in den Hadithen bei Sahih Muslim, Buch 026, Nr. 5430 und Sahih Bukhari, Band 3, Buch 47, Nr. 786.
} 
Religionen akzeptieren müssen. Und als sie Heiden bekämpften, wollte er keinen von ihnen töten, weil er dachte, dass es vielleicht ein Gläubiger sein könnte." (Jalil, 42, arabisch-muslimischer Mann aus Bagdad/Irak). Jalils Aussage entpuppt sich bei genauerem Hinsehen als Scheintoleranz, wenn sie wörtlich auf heutige Verhältnisse übertragen wird, insbesondere gegenüber Atheisten. Yanes war aber auch mit dieser Scheintoleranz nicht einverstanden und warf daraufhin ein: „Aber letztlich werden sie [die Juden; Anm. GJ] ${ }^{35}$ zu unseren Feinden. Laut Koran und dem Islam sagt Gott uns, wer die Feinde deiner Religion sind. Letztlich sagt uns Gott, dass dies deine Feinde sind." (Yanes, 32, arabisch/palästinensisch-muslimischer Mann aus Damaskus/Syrien) Yanes hat durchaus recht, dass es im Koran und auch in den Hadithen (Erzählungen über den Propheten Mohammed) Textstellen gibt, die insbesondere bei einer weitverbreiteten - ahistorischen, nicht kontextualisierten Interpretation nahelegen, dass Jüdinnen und Juden Feinde des Islam und der Muslime schlechthin seien. ${ }^{36}$ Verweise auf die Annahme einer entsprechenden Feindschaft machten auch Interviewte, die diese Ansichten selbst nicht teilten: „Es gibt ein Riesenproblem zwischen dem Islam und Juden. Und dieses Problem stammt aus der Zeit des Propheten.“ (Nidal, 35, kurdischer Mann aus Aleppo/Syrien mit muslimischem Hintergrund). Das in islamistischen Kreisen verbreitete Bild, Juden würden von Affen und Schweinen abstammen, ${ }^{37}$ war vereinzelten Interviewten aus YouTube-Videos von religiösen, öffentlichen Persönlichkeiten bekannt, stieß bei ihnen aber auf Ablehnung.

Als Beweis einer Feindschaft der Juden gegenüber Muslimen diente einigen Befragten die Annahme, Juden hätten versucht, Mohammed zu töten. So berichtete Abia (schätzungsweise Mitte 20, arabisch-muslimische Frau aus Bagdad/Irak) davon, dass die Mehrheit der Menschen im Irak keine Jüdinnen und Juden mag, da sie annähme, dass diese die Werte von Muslimen nicht respektierten und schlecht über den Islam redeten. Hayyan (schätzungsweise Ende 20, arabisch-muslimischer Mann aus Quneitra/Syrien) fügte daraufhin an, „die Juden“ hätten versucht, „den Gesandten“ zu töten. Dieses Gerücht lässt sich zurückverfolgen auf die kanonisierten Hadithe, die Erzählungen über das Leben Mohammeds, in denen es heißt, eine jüdische Sklavin habe Mohammed vergiftetes Fleisch zu essen gegeben. ${ }^{38}$ Islamisten, und in diesem Fall auch einer der

35 Auf Nachfragen des Übersetzers, wer der Feind sei, antwortete Yanes „Israel,“ womit klar wird, dass er nicht Ungläubige im Allgemeinen meint. Da es im Gespräch zuvor hauptsächlich um Juden ging und er an anderer Stelle äußerte, dass er keinen Unterschied zwischen Juden und Israelis sehe und darüber hinaus die Aussage mit dem Bezug zum Koran unsinnig wäre, wenn sie auf Israel und nicht auf Juden bezogen würde, kann geschlossen werden, dass hier Juden gemeint sind.

${ }^{36}$ Besonders relevant unter einer Anzahl von judenfeindlichen Stellen im Koran scheinen: Sure 5, Vers 82, der besagt, dass unter Juden die erbittertsten Gegner der Muslime zu finden sind, weit mehr als unter Christen; Sure 2, Vers 61, der beschreibt, dass Gott die Juden mit einem Fluch belegte; Sure 2, Vers 96, in dem Juden vorgeworfen wird, sie liebten das weltliche Leben; sowie Sure 9, Vers 29, der zum Kampf gegen Juden und Christen aufruft, bis sie Tribut zahlen und sich unterwürfig zeigen. Die kanonischen Hadithe enthalten noch explizitere judenfeindliche Stellen.

${ }^{37}$ Neil J. Kressel, ,The Sons of Pigs and Apes“. Muslim Antisemitism and the Conspiracy of Silence, Dulles 2012.

38 Sahih Muslim, Buch 26, Nr. 5430 und Sahih Bukhari, Band 3, Buch 47, Nr. 786. 
Interviewten, machen daraus den Vorwurf, dass schon seit Zeiten Mohammeds „die Juden“'Todfeinde der Muslime seien.

Einer der wirkmächtigsten Vorwürfe gegen „die Juden“ in der Tradition des Islam - in mancher Hinsicht vergleichbar mit dem Vorwurf im Christentum, „die Juden“ hätten Jesus getötet - ist die Vorstellung, sie hätten die heiligen Schriften gefälscht. Dieser Mythos findet sich an mehreren Stellen auch direkt im Koran. ${ }^{39}$ Damit verbunden sind Stereotype von „den Juden“ als Verrätern und Lügnern.

„Was wissen wir über Juden? Sicher, eine Religion, aber sie haben sie verfälscht. [...] Das wissen wir. Sie haben ein Buch wie unseres und sie haben einen Propheten und wir erkennen ihren Propheten an und alles, aber sie haben das Buch gefälscht, das von Gott offenbart wurde [...]. Der Koran besagt auch, dass es nicht dasselbe Buch ist." (Bader, 33, arabisch/palästinensisch-muslimischer Mann aus dem Flüchtlingslager Jarmuk bei Damaskus/Syrien)

Auch der Wunsch nach der Zerstörung Israels kann religiös begründet werden, indem Palästina, das in diesen Vorstellungen das Gebiet Israels mit umfasst, als muslimisches Territorium deklariert wird, auf dem Juden und damit der jüdische Staat keinen Hoheitsanspruch haben dürfen. ${ }^{40}$

„Letztendlich ist Palästina unser. Wir sind Muslime und wir wissen, dass Palästina den Muslimen und Arabern gehört und Aksa [am Tempelberg in Jerusalem; Anm. GJ] unser ist und eines Tages wird Palästina frei sein und $\mathrm{zu}$ uns zurückkehren." (Yasser, 41, arabisch/palästinensisch-muslimischer Mann aus Homs/Syrien)

Inas und Fatin, zwei junge, säkulare und wenig religiöse Frauen aus Damaskus, erwähnten eine in ihrem Bekannten- und Familienkreis sowie in Schulen und Schulbüchern in Syrien verbreitete Vorstellung: ,Ich habe gehört, im Islam, dass wir vor dem Jüngsten Tag Palästina zurückkriegen und den Krieg gewinnen werden." (Inas, schätzungsweise Mitte 20, arabisch-muslimische Frau aus Damaskus/Syrien). Diese Annahme zeigt die Verflechtung säkularer, islamischer und nationalistischer Deutungen des Krieges gegen Israel beziehungsweise gegen „die Juden“. Sie ist anschlussfähig an die in islamistischen Kreisen weitverbreitete Wahnvorstellung eines globalen Krieges gegen den Islam, der von ,den Juden“ angeführt würde, oder an dem sie zumindest mitwirkten. In dieser Vorstellung gibt es in der Zeit kurz vor dem Jüngsten Gericht einen

39 Theologisch stellt sich im Islam das Problem, dass der Koran als direktes Wort Gottes gilt und dass darin Geschichten aus der Thora beziehungsweise dem Alten Testament wiedererzählt werden, bei denen aber einige, teilweise wesentliche Details anders beschrieben werden. Wenn der Text des Korans als absolute Wahrheit gilt, muss die Thora eine Fälschung dieser Geschichten sein, auch wenn historisch unstrittig ist, dass die Thora lange vor dem Koran entstand und Niederschriften aus vorislamischer Zeit existieren. Der Vorwurf der Fälschung findet sich im Koran, so etwa in Sure 4, Vers 46 oder in Sure 2, Vers 75, in denen Juden vorgeworfen wird, wissentlich Gottes Wort entstellt zu haben.

${ }^{40}$ Eine entsprechende Logik lässt sich auch bei islamischen Organisation in Deutschland verzeichnen, die eine Nähe zum Islamismus zeigen, wie dem eingangs erwähnten Rat der Imame und Gelehrten. Dieser erklärte im Dezember 2017: „Der östliche wie der westliche Teil Jerusalems sind als arabisches muslimisches Land zu betrachten.“ (RiGD, Pressemitteilung vom 7.12.2017.) 
Religionskrieg, aus dem der Islam als Sieger hervorgehen werde. ${ }^{41} \mathrm{Gamal}$, ein ehemaliger Schuldirektor und Hochschullehrer, führte als Beleg für diese Position die „Protokolle der Weisen von Zion“ an, aus denen er frei zitierte: „Wir haben unser Bestes getan, um das Christentum in Europa zu erledigen und es innerhalb der Kirche zu beschränken. [...] Und wir werden nicht lange brauchen, um den Islam zu erledigen." (Gamal, 45, arabisch-muslimischer Mann aus Idlib/Syrien). Den in seinen Augen allgegenwärtigen Vorwurf, „der Islam“ sei die Ursache des Terrorismus, sah Gamal als Bestätigung für die Echtheit der „Protokolle“, die er als jüdisches Buch ansah und von denen er gleich zwei Exemplare besitzt, wie er berichtete.

Einige Interviewte begründeten ihre Abneigung gegenüber Jüdinnen und Juden auch mit muslimischen Traditionen. Inas etwa erinnerte sich an muslimische Verhaltensregeln gegenüber jüdischen und christlichen Menschen. „Was ich höre, ist, dass Juden wie wir sind. Sie sind sauber wie Muslime, [...] aber du darfst nicht in ihrem Haus schlafen, weil $\mathrm{du}$ [dort] nicht sicher bist. Christen sind nicht sauber, aber du kannst in ihrem Haus schlafen. Aber Juden sind unsere Cousins, sie sind wie wir, was Alltagsgewohnheiten betrifft." (Inas, schätzungsweise Mitte 20, arabisch-muslimische Frau aus Damaskus/ Syrien).

\section{Antiisraelismus: Gegen die Existenz Israels}

Negative Einstellungen gegenüber Israel und die Wahrnehmung, dass die Existenz des Staates prinzipiell unrechtsmäßig sei und beendet werden sollte, gehörten für die meisten der befragten Geflüchteten (mit Ausnahme vieler kurdischer) zur Norm. Dazu gehörte, dass Zionismus grundsätzlich negativ konnotiert war. Unter den Begriff Zionismus wurden jedoch auch viele Phantasien über angebliche israelische Einflüsse in der Welt sowie über vermeintliche Expansionspläne Israels gefasst - er wurde also keineswegs nur als Nationalbewegung zur Errichtung eines jüdischen Staates verstanden. Gerade das einfache Schwarz-Weiß-Denken und die Pauschalisierungen in Bezug auf Israel, verdeutlichen, dass es sich hierbei um eine grundsätzliche Opposition gegen Israel handelt - d.h., dass es sich eben nicht um Kritik, sondern vielmehr um ein antisemitisches Ressentiment handelt. Die Vorstellungen der Befragten von Jüdinnen und Juden einerseits sowie von Israel und Israelis andererseits gingen häufig ineinander über - auch bei denen, die eingangs betonten, dass es einen großen Unterschied zwischen Juden und Israelis gebe.

Interviewte, die sich antiisraelisch äußerten, positionierten sich zwar pro-palästinensisch (auch wenn sie keinen palästinensischen Hintergrund haben), ${ }^{42}$ begründeten ihre

${ }^{41}$ Ronald L. Nettler, Past Trials and Present Tribulations. A Muslim Fundamentalist's View of the Jews, Oxford 1987.

42 Interviewte mit palästinensischem Hintergrund hoben ihre palästinensische Identität oft hervor und empfanden es als selbstverständlich, dass mit dieser Identität israelfeindliche Einstellungen einhergehen, ohne dass auf konkrete Leiden von PalästinenserInnen und israelische Verantwortlichkeiten im Einzelnen eingegangen wurde. 
Ablehnung Israel gegenüber aber weniger mit dem Leid der PalästinenserInnen als vielmehr prinzipiell: Allein die Existenz eines jüdischen Staates auf vermeintlich arabischmuslimischem Territorium schien für sie nicht akzeptabel.

Für Gamal, den ehemaligen Schuldirektor und Hochschullehrer, war es eine Selbstverständlichkeit, Israel das Existenzrecht abzusprechen. Auf die Frage des Interviewers „Denken Sie, dass es eine schlechte Idee ist, dass Israel existiert?“ antwortete Gamal mit der knappen Antwort „Ja selbstverständlich.“ Doch dann schob er der Aussage den folgenden, relativierenden Satz hinterher: „Aber nicht schlimmer als Bashar Al Assad.“ (Gamal, 45, arabisch-muslimischer Mann aus Idlib/Syrien) Eine Reihe von syrischen Interviewten betonte, dass die Verbrechen des syrischen Regimes und des IS gravierender sind als die Israel zugeschriebenen Verbrechen. „Israel hat in seiner ganzen kriminellen Geschichte in Palästina nicht so viele Menschen getötet wie Bashar al-Assad in sechs Monaten in Syrien, “ sagte etwa Burhan (27, kurdisch-muslimischer Mann aus Damaskus/Syrien), der keineswegs frei war von antiisraelischen Verschwörungsphantasien. Allerdings ließ sich bei ihm eine Infragestellung des Feindbildes Israel beobachten. Andere Befragte wie Gamal sahen jedoch eindeutig ein Komplott am Werk: Gamal beschrieb Bashar al-Assad als „Partner“ des Islamischen Staates. Die Begründung: Beide würden Israel nicht bekämpfen, obwohl Israel doch Syrien angreife. Gamal vermutete daher, dass alle drei Akteure unter einer Decke steckten. Wieder andere gingen davon aus, dass Syrien angegriffen wird, „um Israel zu schützen." (Zaid, 29, arabisch-muslimischer Mann aus Syrien). Dass Israel hinter den Kriegen in Syrien und im Irak stecke, glaubten interessanterweise sowohl säkulare wie religiöse, christliche wie muslimische, männliche wie weibliche Interviewte. Manche betonten eher den (negativen) regionalen Einfluss Israels und hoben israelische Angriffe auf Syrien und die Besetzung der Golanhöhen oder des Südlibanons hervor, die sie als Teil eines Plans zur Errichtung eines Groß-Israels ansahen, der bis 1948 oder noch früher zurückreiche.

Der Hauptpunkt, mit dem die Befragten ihre antiisraelische Haltung begründeten und bei dem sie von einem allgemeinen Einverständnis auch des Interviewers ausgingen, ist die Verurteilung der „Besatzung Palästinas“, womit allerdings nicht die Besatzung des Westjordanlandes, sondern die Staatsgründung Israels gemeint ist. Dabei kann das vorherrschende Bild von Juden durch entsprechende Propaganda des syrischen oder irakischen Regimes geprägt sein. Dies reflektierte Assad rückblickend für die Zeit, bevor er nach Deutschland gekommen war: „Ich wusste so gut wie nichts über Juden. Alles, was ich wusste, ist, dass die Juden palästinensisches Land besetzen. “ (Assad, 31, arabischmuslimischer Mann aus Homs/Syrien) ${ }^{43}$

\footnotetext{
43 Assad widersprach antisemitischen Verschwörungsphantasien anderer Befragter und zeigte wenig Ressentiments gegenüber Juden. Er glaubte jedoch das Stereotyp, dass Juden reich seien und ihnen ,alles gehört“, von seinen jüdischen Bekannten in Deutschland als wahre Zuschreibung gelernt zu haben.
} 
Andere leugneten selbst die historische Tatsache, dass Jüdinnen und Juden bereits lange vor der Gründung des Staates Israel im Nahen Osten lebten. Juden wurden oft ausschließlich als Europäer und damit als Fremdkörper auf arabischem Territorium angesehen:

Interviewer: „Aber denken Sie nicht, dass Juden bereits dort waren [in Jerusalem und Hebron; Anm. GJ], auch schon vor zweitausend Jahren?“ Yasser: „Wer? Juden? Nein, Juden waren in Großbritannien, Juden sind nicht in Palästina zu Hause. [...] Juden haben kein Zuhause, sie haben kein Land." (Yasser, 41, arabisch/palästinensisch-muslimischer Mann aus Homs/Syrien)

Das Recht auf jüdische nationale Selbstbestimmung verneinte Yasser nicht nur mit dem Argument, dass es keine Geschichte jüdischen Lebens im Nahen Osten gegeben hätte. Ganz prinzipiell postulierte er, dass Jüdinnen und Juden gar kein eigenes Land hätten und griff damit auf das Stereotyp jüdischer Wurzellosigkeit und ewiger Wanderschaft zurück. ${ }^{44}$ Andere Interviewte akzeptierten zwar die historische Tatsache, dass Jüdinnen und Juden seit Jahrhunderten nicht nur in Jerusalem, sondern auch im Irak und in Syrien leben. Aus ihrer Perspektive bestätigte dies aber nur, dass ein harmonisches Zusammenleben am besten unter arabischer beziehungsweise muslimischer Herrschaft möglich ist. Letztlich wird daraus eine Forderung nach Auflösung des jüdischen Staates zugunsten eines muslimisch oder arabisch dominierten Palästinas, in dem Jüdinnen und Juden toleriert werden. Ein Vertreter dieser Position ist Zaid:

„In unserer arabischen Mentalität gibt es keinen Frieden, solange du nicht zurückgibst, was genommen wurde [...]. Was ich will, ist, dass deren Armee zerstört wird. Sie mögen dann weiter auf unserem Gebiet leben und ihre Religion ausüben [...]. Das ist, was wir für die nächsten 25 Jahre wollen, inschallah [so wahr Gott will; Anm. GJ]. Und ich wiederhole es zum dritten Mal, wir werden für die Zukunft der gesamten arabischen Nation entscheiden, auch wenn es einen dritten oder vierten Weltkrieg geben wird, weil wir eine große Generation haben, die in dieser Kultur groß geworden ist. Wir haben kein Problem mit den jüdischen Menschen, wir glauben, dass das [auch] deren Land, deren Region ist, aber wir wollen ein Ende der israelischen Armee und sie wird zerstört werden. Und sie mögen dann mit uns, unter der palästinensischen Regierung, leben und ihre jüdische Religion ohne Probleme ausüben. Wir wollen nur in Frieden und Sicherheit leben." (Zaid, 29, arabisch-muslimischer Mann aus Syrien)

44 Yasser ist ein gutes Beispiel für jemanden, der versuchte, zwischen Juden und Israel zu trennen. Die Fundamentalopposition gegen Israel, das Leugnen eines jüdischen Volkes und seiner Verbindung zu Israel sowie die Verwendung alter judenfeindlicher Stereotype hielten ihn nicht davon ab, an anderer Stelle zu sagen: „Wir sind nicht gegen Juden. Manche sind gut und manche sind schlecht. Netanyahu ist ein schlechter Mensch, aber das sind nicht alle Bürger." (Yasser, 41, palästinensisch-syrischer Herkunft aus Homs/Syrien) - Auch der oben mit einer Verschwörungstheorie in Bezug auf die angeblich von Israel initiierte Zerstörung Syriens zitierte Jaleel stellte an anderer Stelle seine nicht sehr originelle Verschwörungsphantasie zum 11. September 2011 vor, als angeblich 3.000 jüdische Angestellte nicht zur Arbeit erschienen, und ergänzte dann: „Ich habe hier drei sehr nette jüdische Freunde, also das ist in Bezug auf die Regierungen." (Jaleel, 31, Christ aus Damaskus/Syrien) 
„Frieden und Sicherheit“ ist in dieser Vorstellung also nur durch Unterwerfung unter den islamischen Herrschaftsanspruch zu haben. Die fundamentale Ablehnung des jüdischen Staats zeigte sich auch bei einigen sehr westlich orientierten Interviewten darin, dass sie die offizielle Bezeichnung Israel ablehnten und stattdessen den Begriff „Palästina“ verwendeten. Dazu die bereits zitierte Inas: „Mein Traum ist, dorthin zu fahren und Palästina zu sehen. Wirklich, das ist ein Traum von mir.“ Auf Nachfrage des Interviewers, ob sie Israel oder Palästina meine, kam die freundliche, aber bestimmte Antwort: „Nein, nein, das ist Palästina. Palästina und etwas, das Tel Aviv genannt wird.“ (Inas, schätzungsweise Mitte 20, arabisch-muslimische Frau aus Damaskus/Syrien).

Das seit dem Mittelalter im Christentum tradierte antisemitische Stereotyp von Juden als Kindermördern wird in medialen Diskursen in modernisierter Form häufig auf Israel übertragen. In den Interviews wurde nur vereinzelt auf dieses emotionalisierende Bild verwiesen. Das mag daran liegen, dass erstens zum Zeitpunkt der Interviews im Dezember 2016 die mediale Berichterstattung zum Nahostkonflikt kaum entsprechend interpretierbare Bilder lieferte, zweitens einigen die Bilder von an Giftgas erstickten syrischen Kindern noch sehr präsent sein mochten und drittens das Leiden der Palästinenser für viele nicht zentral war in ihrer Ablehnung von Israel. Insgesamt lässt sich beobachten, dass die Interviewten mit palästinensischem Hintergrund dem israelisch-arabischen Konflikt sehr viel mehr Bedeutung beimaßen und diesen auch emotionaler diskutieren als die anderen Befragten. Vielen Interviewten erschien der israelisch-arabische Konflikt angesichts der Kriege in Syrien und im Irak weniger bedeutsam.

Die Frage an die Interviewten, ob sie denken, dass das, was die Nazis mit den Juden gemacht haben, das Gleiche sei wie das, was Israel mit den Palästinensern mache, führte zu einer Reihe höchst unterschiedlicher Reaktionen. Der Vergleich stieß in gleichem Ausmaß auf Zustimmung und Ablehnung. Eine Zustimmung ging oft einher mit einer fundamentalen Ablehnung des Existenzrechts Israels - und scheint vielfach gerade in dieser Ablehnung des Existenzrechts motiviert zu sein, da gar nicht erst versucht wurde, bestimmte Aspekte des Holocausts mit der derzeitigen Situation in Israel zu vergleichen. Eine vage Gleichsetzung der Maßnahmen seitens der israelischen Regierung gegenüber Palästinensern mit der nationalsozialistischen Vernichtung der Juden kann aber auch in Unwissen - sowohl bezüglich des Holocausts als auch des israelisch-palästinensischen Konflikts - und einer generellen Ablehnung von Gewalt begründet sein. „Mord ist Mord“", antworteten viele, wenn sie nach ihrer Meinung zu dem Vergleich zwischen Nazis und Israelis gefragt wurden, was durchaus eine Basis für aufklärerische Bildungsarbeit sein könnte.

Umgekehrt bedeutet die Ablehnung einer Gleichsetzung jedoch nicht notwendigerweise, dass die Staatsgründung Israels grundsätzlich als legitim anerkannt wird - zumindest dann nicht, wenn noch prinzipielle Parallelen gesehen werden. Dila (26, atheistische Kurdin aus Syrien) war der Meinung, dass es ,die gleiche Idee [ist], aber in einer anderen Weise. Die Israelis stahlen das Land, auch wenn sie nicht so viele Menschen umbrachten 
und Hitler so viele Menschen umbrachte.“ Interessanterweise wurde der Vergleich oft von Angehörigen von Minderheiten abgelehnt, das heißt, vor allem von Kurden, aber auch von einigen Christen und Atheisten. Die Ablehnung oder Zustimmung zur Gleichsetzung von Israel mit NS-Deutschland schien weniger vom spezifischen Wissen über den Holocaust und über den israelisch-palästinensischen Konflikt abzuhängen, als davon, ob Ressentiments gegenüber Israel und Juden vorherrschten oder nicht.

Ein arabisch-muslimischer Mann aus Syrien, Hayyan, schätzungsweise Anfang dreißig, hatte einen ganz besonderen Grund, weshalb er die Gleichsetzung von Israel mit den Nazis ablehnte. Er stammt aus Quneitra in den Golanhöhen, direkt angrenzend an Israel. Sein Vater war zwei Jahre in Israel im Gefängnis, wurde dort aber gut behandelt. Und er wusste zu berichten: ,,auch heute, in den Gegenden, die nah an Israel sind, wann immer es verletzte Menschen an der Grenze gibt, nehmen sie [die Israelis; Anm. GJ] sie und geben ihnen medizinische Hilfe, was immer die Behandlung auch kosten mag." Hayyan schien zwar auch nicht mehr über den Holocaust zu wissen als andere Interviewte und er verurteilte Israel auch dafür, sein Heimatdorf (zeitweise) besetzt zu haben, eine Dämonisierung Israels mittels einer Gleichsetzung mit den Nazis hielt er aber dennoch für falsch. Die Einhaltung rechtsstaatlicher Normen in israelischen Gefängnissen und vor allem die israelische Hilfe für syrische Verletzte schienen einen nachhaltig positiven Effekt auf Hayyans Einstellungen zu Israel und Israelis bewirkt zu haben.

\section{Die „reichen Juden“ und Verschwörungsphantasien als Welterklärung}

Das weltweit wohl verbreitetste antisemitische Stereotyp vom reichen Juden kam auch in den Interviews sehr viel häufiger vor als andere Stereotype. ${ }^{45}$ Viele Interviewte nahmen wie selbstverständlich an, dass Juden reich seien. Diese vermeintliche Tatsache bewerteten sie aber nicht unbedingt negativ.

„Die Idee, dass Juden viel Geld haben, ist wirklich normal in unserer Gesellschaft. Und wo ist das Problem, wenn sie viel Geld haben? Syrischen Händlern wurde von jüdischen beigebracht, wie sie ihre Gewerbe managen." (Qadir, 31, arabisch-christlicher Mann aus Damaskus/Syrien)

Einige Befragte formulierten bewusst vorsichtig und zeigten damit ihre Unsicherheit: „Wir Araber denken, dass Juden die reichsten Leute der Welt sind, aber das mag falsch sein." (Maen, 23, arabisch-muslimischer Mann aus Khirbet Ghazaleh/Syrien) Andere hingegen führten vermeintliche Belege von einer angeblichen Dominanz von Juden in Banken oder der Geschäftswelt an. Das Bild von den reichen Juden wurde auch auf Israel

45 Nur in einem Interview sagten zwei syrisch-palästinensische Befragte (Suaad, 27, und Sabri, 41, aus dem Flüchtlingslager Jarmuk bei Damaskus beziehungsweise aus Homs/Syrien), dass sie von dem Stereotyp des reichen Juden noch nie gehört hätten. 
bezogen, eingebettet in Verschwörungsphantasien, in denen Israel und die Juden insgesamt die Welt durch Geld beherrschen:

„Israel, insbesondere Juden, sind bekannt dafür, dass sie die größte Finanzmacht in der Welt sind, also die beherrschen die Welt mit ihrem Geld. Gerade erst gestern habe ich gelesen, dass ein jüdischer Doktor [...] sagte, dass das Star Academy-Programm von uns gemacht wurde und wir es durch Europa in der arabischen Welt verbreiten, um die arabische Frau zu demütigen, weil der arabische Mann schon gedemütigt ist. Als ihm gesagt wurde, dass es das Programm im Libanon gibt, sagte er [der jüdische Doktor; Anm. GJ], dass es von ihnen gesponsert ist, von den Juden, Israel und Amerika. Also die beherrschen die Welt mit ihrem Geld.“ (Ratib, 36, arabisch-muslimischer Mann aus dem Irak)

Ratibs Zitat illustriert, wie das Stereotyp der reichen Juden mit „jüdischer Weltmacht“ assoziiert wird, die angeblich zersetzend und bedrohlich für die eigene Gemeinschaft ist. In unterschiedlichsten Kontexten sahen Interviewte geheime Machenschaften fremder Mächte am Werk. Vielfach - aber nicht immer - vermuteten sie dabei Juden als Drahtzieher. Dies zeigte sich auch bei der Frage nach Erklärungen für die Kriege in den Herkunftsländern der Befragten. Neben (realistischen) Einschätzungen, dass Länder wie Russland und die USA, aber auch der Iran, Saudi-Arabien und die Türkei, heute im Krieg in Syrien und dem Irak ihre Interessen vertreten, gab es Spekulationen zu den Ursachen, die von Verschwörungsphantasien gekennzeichnet sind. Fremde Mächte hätten die Kriege angezettelt, um den Islam zu zerstören, die arabischen Länder auszubeuten, an ihr Erdöl zu kommen, einen Markt für Waffen zu schaffen oder, um die schlauen Köpfe zur Auswanderung zu bewegen, um sie dann in ihren Ländern als Arbeitskräfte ausnutzen zu können. Diese fremden Mächte werden in den USA, Israel und „den Juden“, oder auch in einer ominösen globalen Waffenindustrie gesehen. Diese Erklärungsversuche zeugen von einem Weltbild, das die Ursache für negative Entwicklungen bei übermächtigen anderen sucht. Dabei wurde häufig eine selektive Geschichtsdarstellung bemüht, in der etwa die Aufteilung des Nahen und Mittleren Ostens nach dem Zusammenbruch des Osmanischen Reiches in französische und britische Einflusssphären 1:1 in die Gegenwart fortgeschrieben und als antiarabische beziehungsweise antimuslimische Verschwörung begriffen wurde:

„Einmal habe ich Hillary Clinton gehört, wie sie über das Ende des Sykes-PicotAbkommens sprach, das sich auf die Teilung der arabischen Länder bezieht und die Laufzeit von einhundert Jahren hat [...], sodass im Jahr 2011 die Menschen in arabischen Ländern revolutionieren und nach Ablauf des Abkommens einen anderen Arabischen Frühling hervorbringen. Also ich denke, das ist eine westliche, amerikanische und israelische Verschwörung gegen arabische Länder, um den israelischen Frieden zu erhalten." (Sayid, 20, arabisch-muslimischer Mann aus Hama/Syrien)

In solch einem Geschichtsbild handeln nicht unterschiedliche Akteure mit unterschiedlichen Interessen den Lauf der Dinge aus, sondern große, undurchsichtige Mächte planen den Lauf der Geschichte von langer Hand und über Generationen 
hinweg. Es ist daher nicht verwunderlich, wenn in diesem Denken auch auf die Tradition antisemitischer Verschwörungsphantasien wie die „Protokolle der Weisen von Zion“ zurückgegriffen wurde und „die Juden“ oder stellvertretend Israel beschuldigt wurden, das eigene Land als Spielball zu nutzen. Diese Verschwörungsphantasien stellten einen unhinterfragten Deutungsrahmen dar, mit dem nicht nur Politik und Geschichte, sondern auch beiläufige, nahezu alltägliche Phänomene interpretiert wurden, die als Bedrohung für die eigene Gesellschaft angesehen wurden. So war Burhan (27, kurdischmuslimischer Mann aus Damaskus/Syrien) überzeugt, dass das Computerspiel „CounterStrike“ von bösen Mächten nach Syrien gebracht wurde, um eine neue Generation kaltblütiger Krieger für den derzeitigen Bürgerkrieg heranzuzüchten. Burhan, der stolz ist auf die syrische Kultur, sah mit großem Bedauern auf die Zerstörung seines Landes, für die er den Mossad, den ,internationalen Zionismus“ und die Freimaurer verantwortlich machte. Interessanterweise war Burhans Weltbild zwar von (antisemitischen) Verschwörungsphantasien geprägt, aber an mindestens einer Stelle brüchig. Er war überzeugt, dass Juden die Banken dominieren und dass Juden vom Anschlag auf das World Trade Center am 11. September 2001 zumindest wussten, weil sie an diesem Tag angeblich nicht zur Arbeit erschienen. Als das Gespräch allerdings auf Israel kam, lobte Burhan die israelische Regierung, weil diese sich für ihre Bevölkerung einsetze und humaner sei als das Regime Bashar al-Assads.

In den verschwörerischen Weltbildern waren die Attentate vom 11. September 2001 häufig ein wichtiger Bestandteil, da sie angeblich von den Amerikanern und/oder den Juden zur Legitimierung der Angriffe gegen die Länder des Nahen Ostens, „die Muslime“ oder „den Islam“ orchestriert wurden. Die amerikanische Politik und der amerikanische Präsident wurden als stark abhängig von der jüdischen oder israelischen Lobby betrachtet.

Eine bei syrischen Interviewten verbreitete Verschwörungsphantasie war ein angeblich geheimes Bündnis Bashar al-Assads mit Israel: Das syrische Regime betreibe zwar nach außen hin antiisraelische Propaganda, verhindere aber tatsächlich jegliches effektive Vorgehen gegen Israel, weil es in Wirklichkeit mit Israel unter einer Decke stecke. Die antizionistische Ideologie des syrischen Regimes war bei einigen so stark verinnerlicht, dass sie diese trotz Opposition gegen das Regime nicht hinterfragten.

Es gab aber durchaus auch Anzeichen, dass selbst diejenigen, die krudesten antisemitischen Verschwörungsphantasien anhingen, zum Teil bereit waren, ihre Ansichten zu überdenken. Maen (23, arabisch-muslimischer Mann aus Khirbet Ghazaleh/Syrien) beispielsweise erzählte von dem ihm zu Ohren gekommenen Gerücht, dass Israel oder Juden die meisten Medien in der Welt und dass jüdische Familien die meisten Banken weltweit kontrollierten. Er erwähnte in diesem Zusammenhang „Die Protokolle der Weisen von Zion“" und ein weiteres verschwörungstheoretisches Buch. Im Anschluss daran gab er allerdings zu, dass dieses auf wenig Fakten beruhe und falsch sein könne. Auch die „Erziehung zum Hass gegen Israel“ in Syrien kritisierte er als Propaganda. 


\section{Distanzierung von antisemitischer Propaganda}

Einige Interviewte lehnten antisemitische Stereotype und Vorstellungen explizit ab. In ihrer Ablehnung wurde jedoch oft zum einen deutlich, dass entsprechende judenfeindliche Einstellungen in ihrem Herkunftsland zur gesellschaftlichen Norm und zur staatlichen Propaganda gehören und zum anderen, dass meist nur ein Teil der antisemitischen Vorstellungen hinterfragt wurde.

Die folgenden drei Beispiele von Fatin, Adnan und Maryam zeigen unterschiedliche Aspekte der Distanzierung von antisemitischen Einstellungen seitens der arabischen Befragten. Fatin studierte zum Zeitpunkt des Interviews in Deutschland, vertrat im Allgemeinen sehr säkulare und tolerante Positionen. Sie spricht hervorragend Englisch und gut Deutsch. Sie glaubte zwar, dass Israel hinter den Anschlägen vom 11. September 2001 stecke, aber sie begann, antisemitische Einstellungen zu hinterfragen. Dazu zählten judenfeindliche Stereotype in der Alltagssprache. Außerdem erkannte sie die staatliche Hetze in Syrien gegen Israel und Juden als Propaganda und bemühte sich um eine Trennung von Juden, Israelis und dem Staat Israel. Adnan, 36, hatte in Damaskus eine Filiale eines internationalen Modegeschäfts geleitet. Im Interview hob er hervor, dass man sich in Syrien im Zweifelsfall judenfeindlich zeigen müsse, um nicht in Schwierigkeiten mit dem Regime zu kommen. Er persönlich hege keine Feindschaft gegen Juden und der Koran schreibe, auch wenn er ihn nicht so genau kenne, Respekt gegenüber Juden vor. An anderer Stelle erklärte er jedoch, dass Juden durch die Besetzung Palästinas zu Feinden würden. Maryam, 35, eine arabisch-atheistische Frau mit christlichem Hintergrund aus Damaskus, sagte, „man kann das, was in der Schule gelehrt wurde, dass Juden habgierig sind und viel Geld haben“, nicht für bare Münze nehmen, denn „sie sind auch Menschen“, wie sie in Deutschland in Begegnungen erfahren habe.

Alle drei, Fatin, Adnan und Maryam, sind wenig religiös und kommen aus Damaskus, einer relativ pluralistischen Großstadt. Alle drei betonten, dass sich die syrische Propaganda nicht nur gegen Israel, sondern gegen Juden richtete. Fatin beschrieb außerdem einen Antisemitismus in der Alltagssprache. Räumliche Distanzierung (Fatin), das Wegfallen der Nötigung zum Antisemitismus durch das syrische Regime verbunden mit einem toleranten Islamverständnis (Adnan) und Begegnungen mit Jüdinnen und Juden in Deutschland (Maryam) führten zum Überdenken antisemitischer Positionen.

Unter kurdischen Interviewten waren antisemitische Stereotype und Verschwörungsphantasien generell weniger verbreitet, auch wenn es Gegenbeispiele gibt, wie es einige der oben zitierten kurdischen InterviewtpartnerInnen illustrieren. Wenn Kurden sich antisemitisch äußerten, dann geschah dies häufiger in gemischten arabischkurdischen Gruppen als in ausschließlich kurdischen Gruppen. Zum Teil mag das an einer instrumentellen Verwendung antisemitischer Bilder liegen: Die Anpassung an den herrschenden (antisemitischen) Diskurs ermöglicht es unter Umständen, bezüglich der 
Interessen der kurdischen Minderheit dem herrschenden Diskurs widersprechen zu können. ${ }^{46}$ In rein kurdischen Gruppen zogen einige Kurden Parallelen zwischen ihrem Volk und Juden beziehungsweise Israel aufgrund der Wahrnehmung eines gemeinsamen Feindes („die Araber“ oder die Staaten Irak, Syrien und die Türkei). Andere sahen Parallelen zwischen der Shoah und dem Giftgasangriff Saddam Husseins 1988 auf die kurdisch-irakische Stadt Halabaja und weitere Ortschaften. ${ }^{47}$

Der ehemalige Lehrer Nidal (35, kurdisch-muslimischer Mann aus Aleppo/Syrien) beschrieb nicht nur das Fehlen einer Feindschaft zwischen Kurden und Juden. Er sprach sogar von einer heimlichen Liebe, die sich daran zeige, dass Juden Kurden geholfen hätten. Er sah Parallelen in der Geschichte des kurdischen und jüdischen Volkes Saddam Husseins Giftgasangriffe und die Ermordung tausender Kurden setzte er mit der Shoah gleich. Sein Bild von Juden war eher philosemitisch geprägt: Gott habe sie mit besonderen Fähigkeiten wie Tüchtigkeit und Reichtum ausgestattet. Siwar (26, kurdischatheistischer Mann aus Qamishli/Syrien) beschrieb einen deutlichen Unterschied zwischen Arabern und Kurden. Während Araber den Juden feindlich gesinnt seien und deshalb sogar deren Ermordung befürworteten, teilten Kurden diese Feindschaft nicht. Dabei verwies er auch auf Unstimmigkeiten in syrischen Lehrinhalten in Bezug auf Israel, die zwar die historische jüdische Besiedlung Kanaans beschreiben, aber den heutigen Juden die Besetzung ,arabischen Landes“ vorwerfen würden.

Gleichsetzungen Israels mit dem Nationalsozialismus stießen bei Kurden meist auf Unverständnis. Sie verwiesen auf die völlig unterschiedlichen Formen von Diskriminierungen beziehungsweise Ermordungen. Siyamend erkannte entsprechende Gleichsetzungen wie auch die kollektive Verurteilung aller Juden für Taten der israelischen Armee als eine Ideologie, die ihm in der Schule beigebracht worden war: „Ich weiß, dass es diese Verallgemeinerungen gab, zum Beispiel, wenn wir sahen, dass die israelische Armee schreckliche Dinge tat, dachten wir, dass alle Israelis so sind. Und das ist etwas, was sie uns in den Schulen gelehrt haben, dass jeder Israeli, Jude, schlecht ist. Auch, weil sie einen kleinen Teil von Syrien besetzten, und solche Dinge werden

\footnotetext{
46 Antijüdische und antiisraelische Einstellungen sind, wie sich auch in den oben genannten Beispielen andeutet, zum Teil arabisch-nationalistisch oder muslimisch identitär aufgeladen und bilden die herrschende Norm im Herkunftsland. Sie können somit eine Signalfunktion übernehmen, um die Zugehörigkeit zur Mehrheitsgesellschaft im Herkunftsland anzuzeigen, welche gerade bei Kurden nach wie vor oft in Frage gestellt wird, wie sich anhand einer langen Diskriminierungsgeschichte sowohl in Syrien und im Irak zeigen ließe. Die antisemitische Norm zu hinterfragen und gleichzeitig für separate kurdische Interessen einzutreten, würde einen Kampf an zwei Fronten bedeuten, beziehungsweise umgekehrt signalisiert das Teilen der antisemitischen Norm, dass das Eintreten für kurdische Interessen nicht auf angeblich zersetzenden Machenschaften Israels besteht, wie dies von arabischen Antisemiten behauptet wird. (MEMRI, Arab Writers: Arab World's Opposition To Kurdish Independence Referendum Is Hypocritical (Special Dispatch 7246), 25.12.2017, [https://www.memri.org/reports/arab-opposition-to-kurdish-statehypocritical], eingesehen 28.10 .2018 .)

47 Ein christlicher Teilnehmer, vermutlich mit armenischem Hintergrund, sieht Parallelen zwischen der Shoah und dem Genozid an den Armeniern, was ihn aber nicht daran hindert, antisemitischen Verschwörungsphantasien über die angeblich von Juden dominierten Freimaurer anzuhängen.
} 
besonders hervorgehoben, während du jung bist, dass Israelis Juden sind und schlecht sind." (Siyamend, 22, kurdisch-muslimischer Mann aus Qamishli/Syrien)

Kurden wurden sowohl in Syrien als auch im Irak als Kurden diskriminiert. Die nationalistisch-arabische Ideologie und Praxis der jeweiligen Baath-Partei war auch gegen sie gerichtet. Dies mag ein entscheidender Grund sein, weshalb auch der antizionistischantisemitische Teil der arabisch-nationalistischen Ideologie abgelehnt wurde. Der morgendliche Treueeid im syrischen Schulsystem beinhaltete zumindest unter Hafez alAssad ein Bekenntnis zum Regime, zur arabischen Einheit sowie zum Kampf gegen die Muslimbruderschaft und gegen den zionistischen Imperialismus. Auch kurdische SchülerInnen wurden unter Androhung der Prügelstrafe gezwungen einen Schwur auf die arabische Einheit zu leisten. Die syrische Kurdin Bervian (26) berichtete: „Der wirkliche Feind für uns Schülerinnen und Schüler war nicht die Muslimbruderschaft oder Israel. Selbstverständlich sprachen sie von Imperialismus oder Zionismus, aber wenn sie darüber sprachen, dann sprachen sie auch über die Kurden [...]. Sie verbanden Kurden mit Imperialismus, dass sie Unterstützung bekämen vom Imperialismus oder imperialistischen Ländern. “ Das trug offensichtlich dazu bei, dass viele KurdInnen die Propaganda gegen den ,zionistischen Imperialismus“ nicht sehr überzeugend fanden.

\section{Schlussfolgerungen}

In fast allen Gruppeninterviews wurden deutlich antisemitische Stereotype geäußert. Die Position, die Welt würde von Juden oder Israel kontrolliert, wird oft als normal oder legitim empfunden. Das Welt- und Geschichtsbild vieler Interviewpartner ist geradezu strukturiert durch Verschwörungsphantasien, insbesondere hinsichtlich der Entwicklungen in der Region des Nahen und Mittleren Ostens. Antisemitische Denkweisen waren auch unter denjenigen verbreitet, die betonten, dass sie das Judentum „respektierten“ oder dass das Zusammenleben zwischen Muslimen, Christen und Juden in den Herkunftsländern und in Deutschland unproblematisch sei.

Ein grundsätzlich negatives Israelbild und eine Infragestellung des Existenzrechts Israels ist für fast alle arabischen Interviewten selbstverständlich. Das Feindbild Israel bricht jedoch vereinzelt auf, insbesondere angesichts der Verbrechen des IS und des syrischen Regimes. Kurdische Interviewte äußerten sich zumindest in rein kurdischen Gruppen des Öfteren neutral oder sogar positiv in Bezug auf Israel. Viele Interviewte betonten, dass sie zwischen Juden und Israel unterscheiden. Dies gelingt aber gerade bei einer starken Abneigung gegen Israel nur punktuell. Andere wiederum sahen explizit keinen Unterschied zwischen Israel und „den Juden“.

Auffallend war die Diskrepanz zwischen Angehörigen der Mehrheitsbevölkerung im jeweiligen Herkunftsland und den Angehörigen der jeweiligen ethnischen und religiösen Minderheiten. Insbesondere einige Kurden zeigten deutlich pro-jüdische und proisraelische Einstellungen. Teilweise waren diese jedoch philosemitisch durchsetzt, das 
heißt, „die Juden“ wurden bewundert für ihre Macht und Cleverness. Wie verbreitet Antisemitismus unter syrischen und irakischen Geflüchteten in Deutschland ist, kann jedoch anhand dieser Studie nicht beantwortet werden und muss in weiteren repräsentativen Umfragen erhoben werden.

An vielen Punkten wurde die antisemitische Norm innerhalb der jeweiligen Herkunftsgesellschaft deutlich. Die antisemitischen Vorstellungen - ob aus arabischnationalistischen, antiimperialistischen, islamistischen oder aus allgemein weltverschwörungstheoretischen ideologischen Versatzstücken gespeist - waren jedoch immer wieder brüchig. Bei einigen InterviewpartnerInnen konnten Ansatzpunkte zur Infragestellung dieser Vorstellungen verzeichnet werden.

Abschließend lassen sich sechs Einflussfaktoren erkennen: eine antisemitische Norm im gesellschaftlichen oder sozialen Umfeld im Herkunftsland; Propaganda im Herkunftsland, einschließlich der Schulbildung; alte und neue Medien, in denen insbesondere internationales antisemitisches „Kulturgut“ wie „Die Protokolle der Weisen von Zion“ und ähnliche Verschwörungsphantasien verbreitet werden; ein Palästinensismus, der per Identifikation mit PalästinenserInnen eine automatische Feindschaft gegen Israel und Juden verbindet; eine panarabische Ideologie mit dem Feindbild eines zionistischen Imperialismus sowie Auslegungen des Islam, die Juden zu Feinden des Islam erklären. Die Versatzstücke antisemitischer Ideologien bilden ein Potenzial für antisemitische Handlungen, die bei einer Radikalisierung oder politischen Betätigung mobilisiert werden könnten. 
